\title{
A novel serum based biomarker panel has complementary ability to preclude presence of early lung cancer for low dose CT (LDCT)
}

\author{
Xiaobing Wang ${ }^{1, *}$, Xiuyi Zhi ${ }^{2, *}$, Zhaogang Yang ${ }^{3, *}$, Haimei Tian ${ }^{1}$, Yanfen Li ${ }^{1}$, Mo \\ Li $^{1}$, Wenya Zhao ${ }^{1}$, Chao Zhang ${ }^{1}$, Teng Wang ${ }^{1}$, Jing Liu ${ }^{1}$, Di Shen ${ }^{4}$, Cuining Zheng ${ }^{4}$, \\ Dan Zhao ${ }^{5}$, Sheng Yang ${ }^{6}$, Jun $\mathbf{Q i}^{4}$, Hongwu Xin ${ }^{7,8}$, Alexander Stojadinovic ${ }^{9}$, Itzhak \\ Avital $^{10}$, L. James Lee ${ }^{3}$, Jianyu Rao ${ }^{1}$ and Wei Zhang ${ }^{1}$ \\ ${ }^{1}$ Tumor Marker Research Center, National Cancer Center/Cancer Hospital, Chinese Academy of Medical Sciences and Peking \\ Union Medical College, Beijing, PR China \\ ${ }^{2}$ Department of Thoracic Surgery, Xuanwu Hospital, Capital Medical University, Beijing, PR China \\ ${ }^{3}$ NSF Nanoscale Science and Engineering Center (NSEC), The Ohio State University, Columbus, OH, USA \\ ${ }^{4}$ Laboratory of Clinical Biochemistry, Chinese Academy of Medical Sciences and Peking Union Medical College, Beijing, PR China \\ ${ }^{5}$ Department of Gynecological Oncology, Cancer Institute and Hospital, Chinese Academy of Medical Sciences and Peking \\ Union Medical College, Beijing, PR China \\ ${ }^{6}$ Department of Medicine, Cancer Institute and Hospital, Chinese Academy of Medical Sciences and Peking Union Medical \\ College, Beijing, PR China \\ ${ }^{7}$ The First Peoples' Hospital of Jingzhou City, The First Hospital and Clinical Medical School of Yangtze University, Jingzhou, PR China \\ ${ }^{8}$ Laboratory of Oncology, Center for Molecular Medicine, Medical School, Yangtze University, Huber, PR China \\ ${ }^{9}$ Uniformed Services University of the Health Sciences, Bethesda, Maryland, USA \\ ${ }^{10}$ P8-Medicine Ltd, Sussex, DE, USA \\ *These authors contributed equally to this work
}

Correspondence to: Wei Zhang, email: zhangww1954@126.com

Keywords: lung cancer, biomarker, early detection, MIC-1, screening

Received: February 03, 2017 Accepted: April 14, $2017 \quad$ Published: April 27, 2017

Copyright: Wang et al. This is an open-access article distributed under the terms of the Creative Commons Attribution License 3.0 (CC BY 3.0), which permits unrestricted use, distribution, and reproduction in any medium, provided the original author and source are credited.

\section{ABSTRACT}

Low Dosage Computerized Tomography (LDCT) has been shown to improve early detection of lung cancer and mortality rates in high-risk individuals, which was, however, limited by specifically coverage for heavy smokers and high rates of false positivity. Here, we aim to investigate a novel biomarker for early detection of lung cancer, and further extend to concentrate high-risk subjects for increasing specificity and coverage of LDCT. We performed retrospective blinded evaluation of lung cancer and healthy controls in training and validation cohorts. Macrophage inhibitory cytokine 1 (MIC-1) alone and panel were assessed. Our data showed the sensitivity of MIC-1 was $\mathbf{7 2 . 2} \%$ and $\mathbf{6 7 . 1 \%}$ for lung cancer diagnosis and early diagnosis respectively, at $\mathbf{9 6 . 6 \%}$ specificity, which were significantly higher than Cyfra21-1, NSE CA125, CEA and SCC. At $90 \%$ specificity, the panel of MIC-1, Cyfra21-1, CA125 and CEA provided $89.5 \%$ sensitivity for early diagnosis of lung cancer, which could be used to concentrate the high-risk subjects for further LDCT screening. We conclude that MIC-1 have great capacity in early lung cancer diagnosis. The algorithmic panel of MIC-1, Cyfra21-1, CA125 and CEA could be used to refine the preselection criteria of high-risk subjects, and thus might facilitate the widespread implementation of LDCT screening.

\section{INTRODUCTION}

Lung cancer is one of the most common human malignant tumors worldwide with considerable attendant societal costs $[1,2]$. Early detection and treatment of lung cancer stands for an urgent global healthcare need and a formidable challenge in the control of this complex and deadly disease [3]. The National Lung Screening Trial concluded specificity of $73.4 \%$ and sensitivity of $93.8 \%$ for annual low-dose CT screening in high-risk smoker [4]. 
However, this type of scan is accompanied by high falsepositive rates and limited applicable coverage. In addition, cost, concerns regarding overdiagnosis, and cumulative radiation exposure remain points of concern. Affordable noninvasive testing such as blood-based biomarkers could potentially improve the positive predictive value of precise LDCT screening, and more importantly, concentrate the potential risk subjects which may in turn extend the applicable coverage for screening potential subjects, such as the non-smoking potential risk subjects.

Although recent advances in molecular diagnostics have generated many candidate biomarkers for lung cancer, the seromarkers developed thus far have not been recognized as ideal biomarkers due to the limited sensitivity and specificity either singularly or as a panel of markers $[5,6]$. The traditional lung cancer biomarkers remain the most studied biomarkers for lung cancer, especially in developing countries [7], which show an increased rate of positivity as the cancer stage advances, but could hardly be serve as standalone indicators of disease at its earliest stages. For example, the most sensitive Cyfra21-1 alone had a reported sensitivity threshold of $44 \%[8,9]$, and the most sensitive early diagnosis of lung cancer methodology with a biomarker panel remains to be determined, though preliminary analysis suggest a relevant sensitivity threshold of $>60 \%$ $[10,11]$. Therefore, new serologic biomarkers with sufficient sensitivity to reliably diagnose asymptomatic patients with lung cancer should be investigated.

Growing evidence indicates that abnormal immune response is involved in cancer patients before clinical confirmation of disease $[12,13]$. Therefore, investigating the associated cytokines involved with the immune response to the developing cancer may be a promising approach to identify biomarkers that can detect cancer at an early stage [14]. Macrophage inhibitory cytokine 1 (MIC-1/GDF15) was originally discovered in macrophage cells and associated with immune inhibition [15-17]. Serum MIC-1 is substantially increased in disease states caused by inflammation and invasive malignancy [18-21]. Numerous studies have demonstrated that MIC-1 plays a valuable function in the biology of carcinogenesis [22-24]. This study aims to explore the value of MIC-1 as a biomarker in the onset of lung cancer, and furthermore, using MIC-1 as a critical factor, construct predictive panel for the preselection of potential risk subjects, which may facilitate the widespread implementation of LDCT screening and enhance its cost-effectiveness.

\section{RESULTS}

\section{Serum MIC-1 is elevated earlier and more progressively than any available clinical biomarkers in lung cancer}

First, we assessed the serum levels of MIC-1 in training group. MIC-1 level was significantly increased in lung cancer patients compared to healthy controls $(p<0.001$; Figure 1 and Supplementary Table 1$)$. Notably, high levels of MIC-1 was observed in early-stage (Stage I/II) lung cancer patients $(p<0.001)$. Moreover, when all patients with lung cancer were grouped according to TNM classification, the gradual increasement in serum MIC-1 levels was evident $(P=0.042)$, with higher levels in advanced patients compared with early-stage patients (Table 1), implying the positive correlation of MIC-1 with lung cancer progression. Further analysis showed that the level of serum MIC-1 was significantly higher in low grade lung cancer, distant metastasis than in high grade tumors or in the absence of distant metastasis, respectively (Table 1).

We also compared MIC-1 with other previously investigated lung cancer serum biomarkers. Although serum from patients with lung cancer showed elevated levels of Cyfra21-1, NSE, CA125, CEA and SCC compared with the healthy group (Supplementary Table 1), our data showed that as single markers, MIC-1 was found to be elevated much earlier stage in the course of the disease and more progressively than any other five clinically available biomarkers. The distributions of all six biomarkers in lung cancer (early-stage lung cancers listed separately), and healthy controls were showed and compared using boxplots (Figure 1). The result from the validation group confirmed these findings, and also showed that serum MIC-1 levels represented a stepwise increasement in benign diseases and lung cancers in comparison with healthy controls, indicating that the overexpression of MIC-1 started likely at the development of lung cancer (Figure 1; Table 1).

\section{Serum MIC-1 significantly improves lung cancer diagnosis, especially early stage diagnosis}

Next, we generated receiver operating characteristic curves (ROC) to evaluate the utilization of the serum MIC-1 protein as a non-invasive diagnostic marker for lung cancer. In training group, the obtained ROC curve of MIC-1, Cyfra21-1, NSE, CA125, CEA and SCC for lung cancer and early-stage lung cancer are graphically shown in Figure 2. MIC-1 had the greatest ability to distinguish lung cancer cases from healthy subjects. The area under ROC curve (AUC) of MIC-1 was 0.962 for all cases, whereas the AUCs of Cyfra21-1, NSE, CA125, CEA and SCC were 0.847, 0.806, 0.787, 0.756 and 0.682, respectively. MIC-1 had significant discriminatory value, especially when only early-stage (Stage I/II) lung cancer samples were tested. The AUC of MIC-1 was 0.953 for early-stage cases, whereas the AUCs of Cyfra21-1, NSE, CA125, CEA and SCC were 0.798, 0.741, 0.689, 0.684 and 0.669 , respectively. These data suggest that serum MIC-1 can significantly improve lung cancer diagnosis, especially early diagnosis.

We further calculated the detection sensitivity of MIC-1 for all lung cancer and early stage I/II lung cancer at various specificity cut-offs (Supplementary Table 2). 
Biomarker distribution
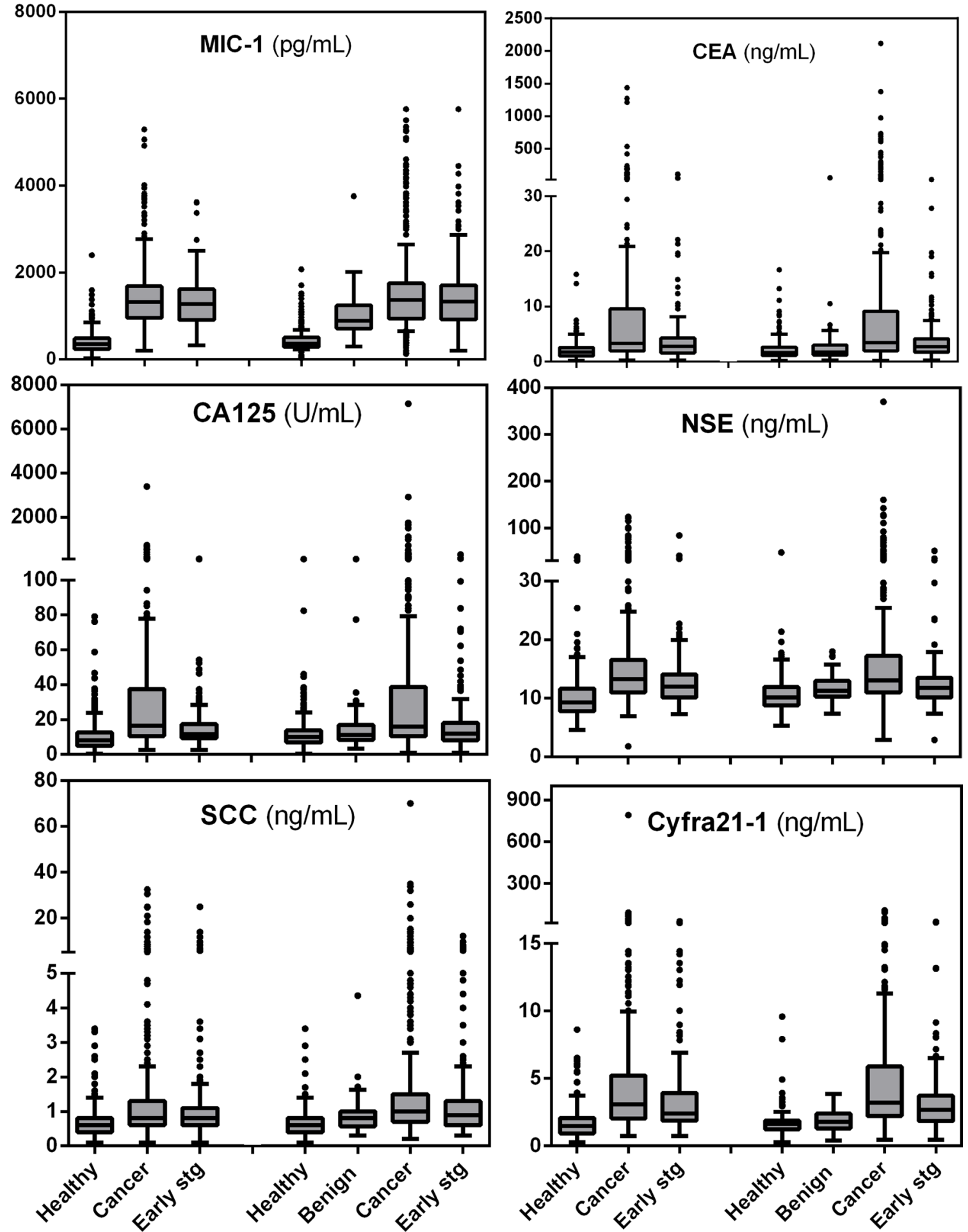

training set

validation set

training set

validation set

Figure 1: Boxplot display of biomarker distributions for controls, lung cancer cases, the subset of early stage (I and II) cases and benign conditions, by training and validation set assignment. Horizontal box boundaries and midline denote sample quartiles. Whiskers mark *adjacent values: upper adjacent value $=$ largest marker value $\mathrm{x}$ such that $\mathrm{x}<=75$ th percentile $+1.5 *$ interquartile distance. Similarly, lower adjacent value $=$ lowest marker value such that $\mathrm{x}>=25$ th percentile $-1.5 *$ interquartile distance. The interquartile distance $(\mathrm{IQR})=75$ th -25 th percentile. 
Table 1: Correlation between MIC1 serum level and clinicopathological variables of lung cancer

\begin{tabular}{|c|c|c|c|c|c|c|c|c|c|c|c|c|c|c|}
\hline \multirow[b]{2}{*}{ Variables } & \multicolumn{7}{|c|}{ Training set } & \multicolumn{7}{|c|}{ Validation set } \\
\hline & $N$ & $\min$ & $\max$ & Median & Mean & IQR & $P$ & $N$ & $\min$ & $\max$ & Median & Mean & IQR & $P$ \\
\hline \multicolumn{15}{|l|}{ TNM stage } \\
\hline stage I & 95 & 319.3 & 3618.0 & 1274.5 & 1348.3 & 766.3 & 0.042 & 123 & 195.2 & 5759.0 & 1245.4 & 1359.6 & 757.2 & 0.017 \\
\hline stage II & 60 & 347.8 & 2465.9 & 1222.0 & 1249.5 & 731.5 & & 76 & 397.5 & 4272.7 & 1355.0 & 1556.1 & 940.1 & \\
\hline stage III & 100 & 396.6 & 5060.1 & 1365.9 & 1551.8 & 792.2 & & 112 & 269.2 & 4493.4 & 1317.2 & 1454.6 & 810.0 & \\
\hline stage IV & 95 & 196.6 & 5292.4 & 1384.9 & 1600.4 & 644.0 & & 100 & 126.0 & 5506.1 & 1477.8 & 1849.6 & 852.7 & \\
\hline \multicolumn{15}{|l|}{$\mathrm{T}$ classification } \\
\hline$T_{1-2}$ & 226 & 317.3 & 5292.4 & 1272.3 & 1342.2 & 686.3 & 0.0004 & 279 & 195.2 & 5759.0 & 1324.5 & 1493.2 & 787.6 & 0.35 \\
\hline $\mathrm{T}_{3-4}$ & 116 & 196.6 & 5060.1 & 1470.0 & 1685.1 & 788.3 & & 120 & 126.0 & 5277.8 & 1405.5 & 1580.2 & 833.2 & \\
\hline Unkown & 8 & & & & & & & 12 & & & & & & \\
\hline \multicolumn{15}{|l|}{$\mathrm{N}$ classification } \\
\hline $\mathrm{N}_{0}$ & 115 & 319.3 & 3770.2 & 1294.9 & 1395.0 & 760.3 & 0.87 & 161 & 195.2 & 5759.0 & 1245.4 & 1380.0 & 743.2 & 0.182 \\
\hline $\mathrm{N}_{1-3}$ & 224 & 196.6 & 5292.4 & 1326.1 & 1471.7 & 726.3 & & 233 & 126.0 & 5506.1 & 1384.0 & 1590.6 & 838.1 & \\
\hline Unkown & 11 & & & & & & & 18 & & & & & & \\
\hline \multicolumn{15}{|l|}{ Remote metastasis } \\
\hline $\mathrm{M}_{0}$ & 255 & 319.3 & 5060.1 & 1276.2 & 1405.1 & 742.1 & 0.039 & 311 & 126.0 & 5759.0 & 1315.9 & 1430.7 & 788.1 & 0.0006 \\
\hline $\mathrm{M}_{1}$ & 95 & 196.6 & 5292.4 & 1395.6 & 1599.7 & 681.7 & & 100 & 151.7 & 5506.1 & 1488.5 & 1884.2 & 858.8 & \\
\hline \multicolumn{15}{|l|}{ Differentiation } \\
\hline Poorly & 140 & 196.6 & 5292.4 & 1347.4 & 1522.7 & 713.1 & 0.072 & 181 & 126.0 & 5506.1 & 1465.0 & 1660.1 & 830.1 & 0.0001 \\
\hline Moderately & 169 & 380.9 & 3773.7 & 1336.3 & 1439.6 & 694.8 & & 161 & 269.2 & 5354.4 & 1310.9 & 1532.3 & 775.2 & \\
\hline Well & 41 & 352.5 & 5060.1 & 1006.9 & 1312.6 & 671.6 & & 69 & 195.2 & 5759.1 & 1092.8 & 1249.3 & 800.6 & \\
\hline \multicolumn{15}{|l|}{ Pathological type } \\
\hline SCLC & 31 & 468.5 & 3680.0 & 1338.5 & 1500.4 & 725.7 & 0.953 & 40 & 126.0 & 4450.7 & 1401.7 & 1603.6 & 749.6 & 0.665 \\
\hline NSCLC & 319 & 196.6 & 5292.4 & 1319.0 & 1453.8 & 731.7 & & 371 & 151.7 & 5759.0 & 1365.6 & 1534.3 & 825.5 & \\
\hline Adenocarcinoma & 114 & 319.3 & 3773.7 & 1381.3 & 1471.8 & 723.3 & 0.182 & 114 & 151.7 & 4050.6 & 1369.7 & 1413.6 & 714.6 & 0.756 \\
\hline Squamous cell carcinomas & 197 & 196.6 & 5292.4 & 1276.6 & 1441.7 & 725.4 & & 247 & 195.2 & 5759.0 & 1347.8 & 1579.63 & 863.9 & \\
\hline others & 8 & & & & & & & 10 & & & & & & \\
\hline
\end{tabular}

For facilitating clinical application, we set $1,000 \mathrm{pg} / \mathrm{ml}$ as the cutoff value for warranting acceptable specificity. The sensitivity of MIC- 1 were $72.2 \%$ and significantly higher than that of Cyfra21-1 (46.6\%), NSE (20.0\%), CA125 (26.6\%), CEA (37.7\%) and SCC (21.1\%), with comparable specificity (Supplementary Table 3). Notably, the sensitivity was $67.1 \%$ in detecting early-stage lung cancer, which was significantly higher than that of Cyfra21-1 (34.2\%), NSE (6.5\%), CA125 (5.8\%), CEA $(20.6 \%)$ and SCC (15.5\%). These data further show that serum MIC-1 could be used for lung cancer detection, especially early lung cancer.

To validate the findings above, we performed similar ROC analysis in the validation group with the healthy individuals as the control group. The results obtained with the training and the validation groups were in good agreement (Figure 2 and Supplementary Table 3). On the other hand, as shown in Supplementary Table 2, when patients with benign conditions were used as the control population in the validation group, the sensitivity of MIC-1 for detecting early stage lung cancer was $65.3 \%$, which is significantly superior to Cyfra21-1 (31.2\%), NSE (3.5\%), CA125 (9.5\%), CEA (17.6\%) and SCC (20.6\%). We further performed ROC curve analysis in subgroups of patients, stratified by histology against the control healthy group. A summary of the AUCs for each of the subgroups is shown in Supplementary Table 4, showing that the associated AUCs are generally similar across all subgroups. Thus, we concluded that MIC-1 has similar discriminating ability for all of the major histological subtypes of lung cancer.

The four biomarker panel MIC-1, Cyfra21-1, CA125 and CEA improves lung cancer diagnosis, especially early diagnosis, more than MIC-1 alone

To further improve diagnostic sensitivity, we used logistic regression on raw values of each marker to establish a model with the above-mentioned serological biomarkers. Linear regression $p$-values were calculated to evaluate whether a single marker of the panel significantly increases the differences between cases and controls, which was described in Supplementary Table 5. A four-biomarker panel consisting of MIC1, Cyfra21-1, CA125 and CEA demonstrated superior performance compared with other combination (Supplementary Table 6). Therefore, we fit an algorithm model using results from the training groups: Logit 
[probability of lung cancer] $=-7.7157+0.005752 *$ MIC-1 + 0.6275*Cyfra21-1 + 0.03770*CA125+ $0.1101^{*} \mathrm{CEA}$. In the ROC analysis, the four-biomarker panel consisting of MIC-1, Cyfra21-1, CA125 and CEA provided a significant improvement in both sensitivity and specificity. At a cut-off probability value of 0.559 , this four-biomarker panel provided $90.6 \%$ sensitivity (95\% CI, 87.0 to $93.4 \%$ ) for lung cancer at $95 \%$ specificity (Figure 3). Furthermore, the panel of fourbiomarker represented comparable classification for the three most common histologic types of lung cancer: Adenocarcinomas (sensitivity, 90.4\%), squamous cell carcinomas (sensitivity, 92.1\%) and SCLC (sensitivity, $83.9 \%$ ). With this unique four-biomarker panel, we were also able to detect both NSCLC and SCLC tumors.
In the analysis of patients with early-stage lung cancer, the four-biomarker panel including MIC-1 identified a sensitivity of $83.9 \%$ at a specificity of $95 \%$; notably, the sensitivity for early stage lung cancer by the four-biomarker panel would increase up to $92.9 \%$ $(95 \% \mathrm{CI}, 87.7 \%$ to $96.4 \%)$ when the specificity was decreased to $90 \%$, at a cut-off probability value of 0.267 .

\section{The significant improvement of lung cancer diagnosis, especially early diagnosis, by the four biomarker panel is validated}

We then analyzed the performance of the predictive model in the validation group, to determine whether the four-biomarker panel could differentiate normal from
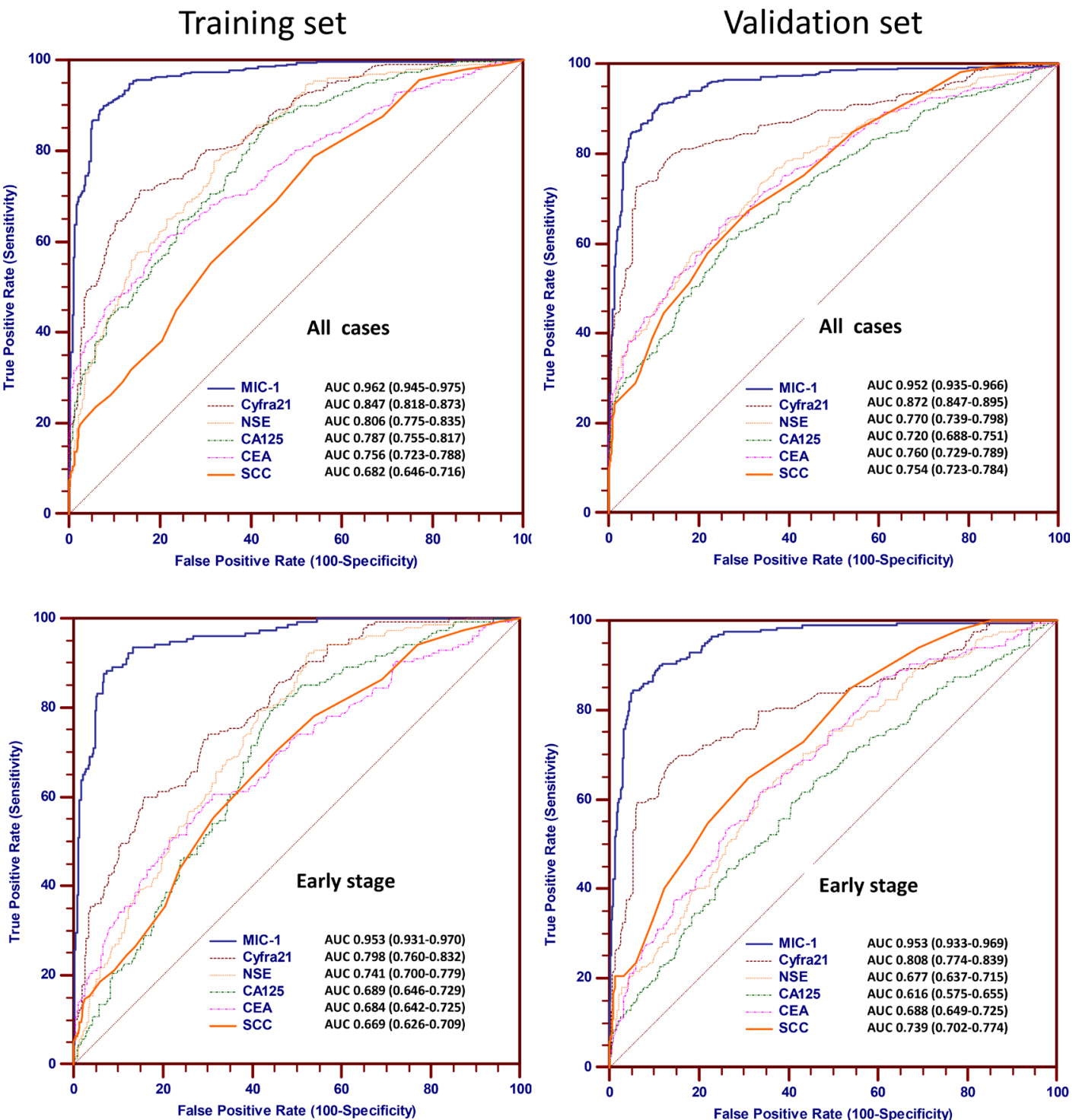

Figure 2: Biomarker ROC curves for cases and controls assigned to the training group and validation group. ROC curves of MIC-1, Cyfra21-1, NSE, CA125, CEA and SCC for discriminating between lung cancer and control in the training group (upper left) and validation group (upper right). ROC curves of MIC-1, Cyfra21-1, NSE, CA125, CEA and SCC for discriminating between early-stage lung cancer and control in the training group (lower left) and validation group (lower right). 
lung cancer samples in the independent group. The panel of four-biomarker conducted well for characterization of patients with lung cancer, offering 0.974 AUC and $90.3 \%$ sensitivity ( $95 \% \mathrm{CI}, 87.0 \%$ to $93.0 \%$ ) at $95 \%$ specificity, which was both significantly higher than MIC-1 alone and the other three biomarker combination tested (Figure 4A). In the analysis of patients with early-stage lung cancer and the healthy subjects used as the control, the panel showed 0.957 AUC, $84.4 \%$ sensitivity ( $95 \%$ CI, $78.6 \%$ to $89.2 \%$ ) at $95 \%$ specificity, and $89.5 \%$ sensitivity $(95 \%$ CI, $84.3 \%$ to $93.3 \%$ ) at $90 \%$ specificity, which is comparable to the $92.6 \%$ sensitivity at $90 \%$ specificity from the training group (Figure 4B). The result from validation group testing further confirmed and demonstrated the superior performance of the four-biomarker panel for lung cancer, especially for early-stage lung cancer.

The performance of the final predictive model for early-stage lung cancer was evaluated using the previously identified cutoff value 0.267 for $90 \%$ specificity of the training group. The sensitivity and specificity of the fourbiomarker panel was $88.4 \%$ and $93.1 \%$, respectively. For 78 samples obtained from patients diagnosed with benign conditions, this four-biomarker panel misclassified 56.4\% blinded benign samples as cancer, more specifically, $52.1 \%$ benign tumor, $57.9 \%$ lung inflammation and $54.5 \%$ pulmonary tuberculosis samples were misclassified as cancer (Figure 4C). The four-biomarker panel provide inferior sensitivity results in the validation group (88.4\%) compared with the training group $(92.6 \%)$, whereas the specificity increased from $90 \%$ to $93.1 \%$, indicating that there may be some inherent population-based differences, and consequently, the cutoff of probability for $90 \%$ specificity could be optimized. This difference could be further refined in planned large scale multicenter studies.

\section{DISCUSSION}

Lung cancer is well-known as a 'silent killer'. Early signs and symptoms, if any, are indistinct and non-specific, and the majority of patients appear with advanced disease. Advanced lung cancer prognosis is dismal, despite aggressive therapy [2, 3, 25]. However, there is currently no good candidate marker for the early detection of lung cancer. In this study, we evaluated MIC-1 as a candidate novel seromarker for the detection of earlystage lung cancers. To our knowledge, the present research is the first in-depth exploration into the potential clinical significance of MIC-1 as a biomarker in lung cancer. We analyzed serum MIC-1 in patients with lung cancer, and demonstrated the diagnostic significance of MIC-1 and the combination of MIC-1 with clinically available biomarkers to discriminate normal tissue from lung cancer, especially early-stage I/II lung cancer with high sensitivity without compromising specificity.

First, in the retrospective research reported here, we have discovered that serum MIC-1 was increased from the early stage and correspond with progression of lung cancer. The elevations of MIC-1 level in patients with early-stage lung cancer are not well understood, since significant levels of tumor-associated products were usually detected in serum at advanced stages of tumor development. However, the known over-expression of MIC-1 by endothelial cells and macrophages in response to inflammatory signals suggests that MIC-1 may act
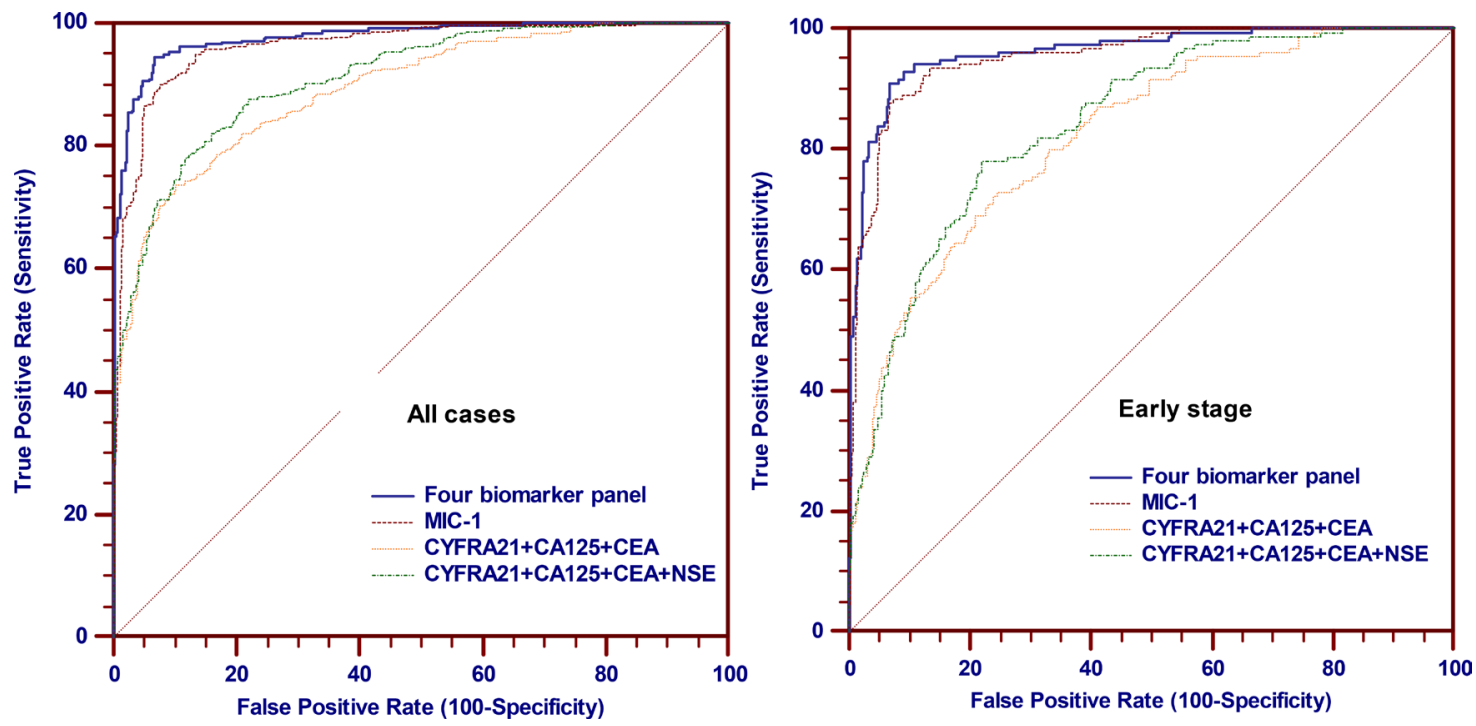

Figure 3: Performance of MIC-1 combining with other biomarkers in the detection of lung cancer in the training group. (Left) ROC curves of four biomarker panel, MIC-1 alone, Cyfra21-1, CA125, CEA combination and Cyfra21-1, CA125, CEA, NSE combination for all lung cancer cases $(N=350)$ and controls $(N=350)$; (Right) ROC curves of four biomarker panel, MIC-1 alone, Cyfra21-1, CA125, CEA combination and Cyfra21-1, CA125, CEA, NSE combination for early-stage lung cancer cases $(N=155)$ and controls $(N=350)$. 
as an important cytokine and play a specific function in inflammatory and immune responses to tumor formation [26-28]. In addition, MIC-1 may act as paracrine/ autocrine cytokine or circulating hormone to stimulate/ inhibit the formation/remodeling of new blood vessels $[22,29,30]$. The growth of new blood vessels, known as angiogenesis, is an important component of tumorgenesis and further uncontrolled tumor growth and metastasis. Therefore, elevated levels of the serum MIC-1 may exert an important action in growth/progression of lung cancer.
Taking these factors into consideration, MIC-1 could be a promising candidate biomarker to detect early stages of the disease through its elevation in the inflammatory microenvironment of the tumor. Further analysis on the value of MIC-1 in the development of lung cancer and early detection of early stage cases is warranted.

In our study, we also compared the distributions of MIC-1 with other biomarkers, Cyfra21-1, NSE, CEA, CA125 and SCC, which are commonly used as lung cancer biomarkers in clinic [31]. Our comparative

B

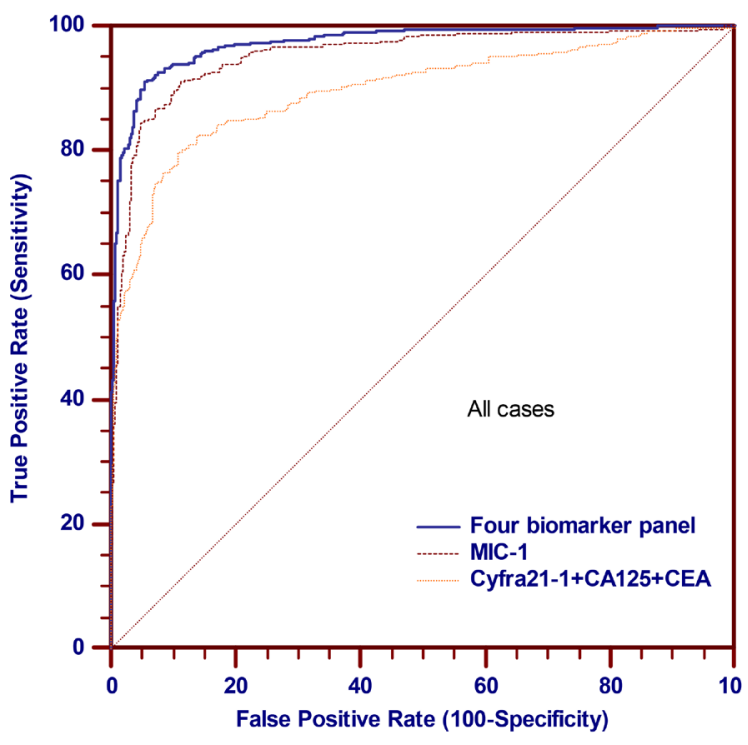

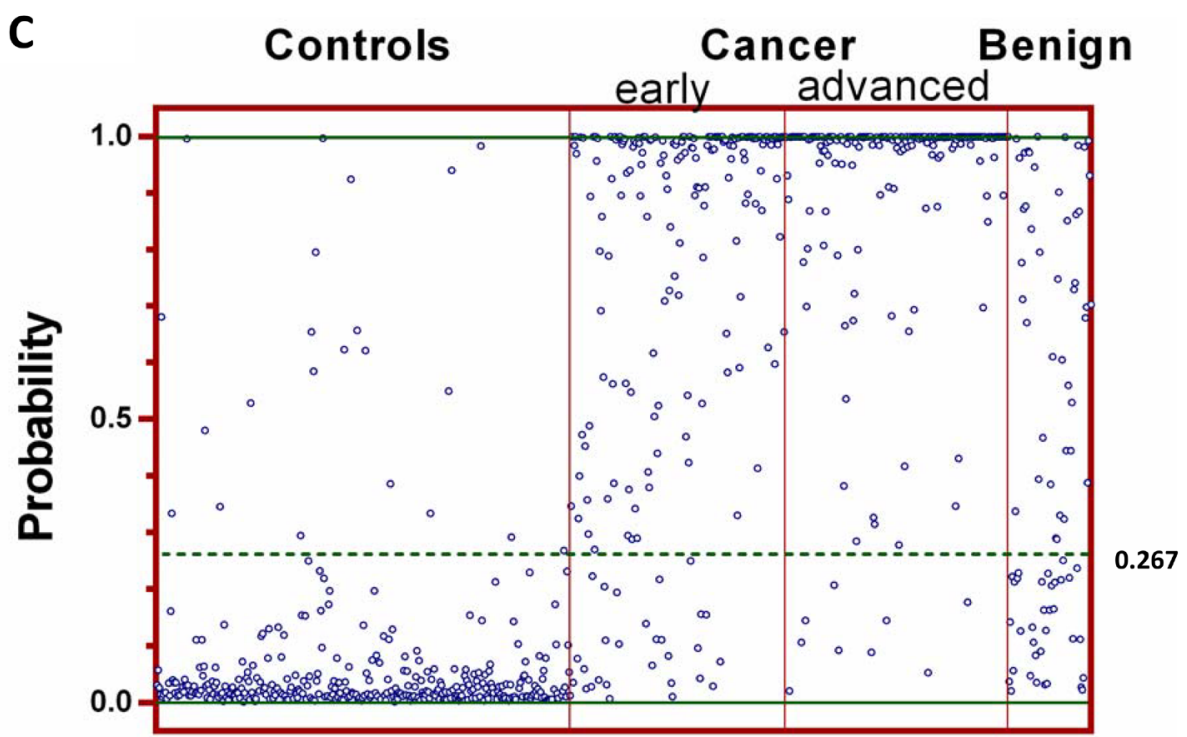

Figure 4: Performance of the four biomarkers panel (MIC-1, Cyfra21-1, CA125 and CEA) in the validation group. (A) ROC curves of four biomarker panel, MIC-1 alone and Cyfra21-1, CA125, CEA combination for all lung cancer cases in the validation group. (B) ROC curves of four biomarker panel, MIC-1 alone and Cyfra21-1, CA125, CEA combination for early-stage lung cancer cases in the validation group. (C) scatterplot of the model predicted disease probabilities by disease group for subjects in the validation group. 
analysis further demonstrates the diagnostic potential of MIC-1 for lung cancer, as its ROC performance was significantly better than the other five markers tested. As a single marker, MIC-1 is the most promising candidate of the six biomarkers tested, especially in early-stage disease. Consequently, the use of MIC-1 could enhance the potential of treating lung cancer in its early stages and this could translate into improved cancer outcomes. Additionally, we tested the above six serum biomarkers in an independent (validation) cohort of lung cancer patients and found that the sensitivity was slightly different. Differences in patient populations tested may explain the subtle differences observed in sensitivity within the training and validation groups. These results clearly imply that MIC-1 could be served as a much more clinically potential biomarker than the other five clinically available biomarkers for early detection of lung cancer.

Numerous results have concluded that the formation of tumor is associated with chronic inflammation [32]. Both lung cancer cells and inflammatory cells secreting MIC-1 may illustrate the higher levels of MIC-1 detected in lung cancer patients. Our data showed that MIC-1 levels in benign disease subjects were significantly higher than healthy controls; while significantly lower than lung cancer group. Considering that elevated serum MIC-1 were detected in subjects with benign diseases and the imaginable interference of the chronic inflammation, we assessed MIC-1 levels in a group of benign disease subjects to study whether MIC-1 can differentiate earlystage lung cancer and non-cancerous conditions including benign lung tumor, pulmonary tuberculosis and lung inflammation. Our ROC results showed that serum MIC-1 was insufficient to differentiate patients with early-stage lung cancer from benign lung tumors, although AUC value was found to approximate that of Cyfra21-1 and to be superior to NSE, CA125, CEA and SCC.

Although the use of MIC-1 alone indicated a promising future for the early diagnosis of lung cancer, we continued to investigate the development of biomarker panels involving this highly sensitive MIC-1, further increasing the performance of early diagnosis. With the use of a logistic regression model, we found that $\mathrm{MIC}-1$ and the other three biomarkers together (MIC-1, Cyfra21-1, CA125 and CEA) are able to reliably discriminate lung cancer samples and healthy control samples. Combinations of these biomarkers could improve diagnostic sensitivity which is preferable to the sensitivity of each of the four traditional biomarkers alone, while maintaining the high diagnostic specificity. We therefore have characterized a four-marker panel (MIC-1, Cyfra21-1, CA125 and CEA) that allows classification of lung cancer with wonderful sensitivity and specificity. This multi-marker panel may act as a prototype of initial screening for asymptomatic subjects. Moreover, our data further indicate that MIC-1 is equally effective in NSCLC and SCLC, as well as with adenocarcinomas and squamous cell carcinomas.
The findings were validated in an independent validation group analysis. Although further analyses of more cases are needed to verify and expand upon the present data, our results strongly suggest that the complement of $\mathrm{MIC}-1$ to the current lung cancer biomarkers may greatly improve the detection sensitivity for lung cancer where these markers are currently utilized clinically.

Currently, studies for early detection are mainly concentrated on the screening of preselected high-risk subjects. These mainly composed of populations with specific genetic predisposition for the formation of lung cancer, along with epidemiological information, such as heavy smoking history [33-35]. It has been suggested that a method with sensitivity and specificity above $90 \%$ would benefit high-risk subjects [36]. Our study has demonstrated that the sensitivity of the four biomarker panel for earlystage lung cancer was $92.9 \%$ and $89.5 \%$ in training and validation group, respectively, with specificity at $90 \%$ for both settings. Therefore, screening for patients with lung cancer would likely need two "steps" to concentrate the population for implement of cost-effective screen. The first step could be epidemiological factor analysis and the second step could be this non-invasive four-biomarker panel. The panel may be used as a 'filter' to identify high-risk subjects, which may in turn improve the positive predictive value of precise screening tools in cost-effective way.

However, it also should be cautious that lung cancers could be revealed with a panel of MIC-1 and the three other biomarkers, while not all subjects detected with this means would suffer from lung cancer, since MIC-1 and the combination values are elevated in patients with benign diseases. Based on the condition that the cutoff was assigned to exclude healthy individuals, any subjects with positive test result were likely to be in unhealthy condition that at a minimum would need additional medical assessment. In such cases, the predictive model should be regarded as helpful for the subjects, no matter which disease was involved. In conclusion, this research presents the framework for the construction of a clinically relevant strategy that could supply a cost-effective and sensitive method to detect early stage lung cancer with the aim of reducing disease-associated mortality.

\section{MATERIALS AND METHODS}

\section{Serum samples and study design}

Serum from 350 lung cancer patients and 350 healthy subjects were collected as the training group from January 2008 to March 2010. Additional independent serum samples from 411 lung cancer patients, 78 benign patients (48 cases of benign tumor, 19 cases of Lung inflammation, and 11 cases of pulmonary tuberculosis) and 389 healthy subjects were collected as the validation group from January 2011 to March 2014. All samples were collected from the Cancer Hospital, Chinese Academy of 
Table 2: Clinical characteristic of the studied subjects

\begin{tabular}{|c|c|c|c|c|c|c|c|c|c|c|c|c|c|c|c|c|c|c|}
\hline \multirow{3}{*}{ Histology } & \multicolumn{9}{|c|}{ Training group } & \multicolumn{9}{|c|}{ Validation group } \\
\hline & \multirow{2}{*}{ No.of Patients } & \multicolumn{3}{|c|}{ Age (years) } & \multicolumn{2}{|c|}{$\begin{array}{l}\text { Sex(No.of } \\
\text { Patients) }\end{array}$} & \multicolumn{3}{|c|}{$\begin{array}{l}\text { Grade (No.of } \\
\text { Patients) }\end{array}$} & \multirow[t]{2}{*}{$\begin{array}{l}\text { No.of } \\
\text { Patients }\end{array}$} & \multicolumn{3}{|c|}{ Age (years) } & \multicolumn{2}{|c|}{$\begin{array}{l}\text { Sex(No.of } \\
\text { Patients) }\end{array}$} & \multicolumn{3}{|c|}{$\begin{array}{l}\text { Grade (No.of } \\
\text { Patients) }\end{array}$} \\
\hline & & range & median & mean & male & female & 1 & 2 & 3 & & range & Median & mean & male & female & 1 & 2 & 3 \\
\hline Healthy controls & 350 & $20-80$ & 59 & 57.7 & 162 & 188 & & & & 389 & $19-78$ & 58 & 57.9 & 205 & 184 & & & \\
\hline Benign lung disease & & & & & & & & & & 78 & $22-75$ & 57 & 56.6 & 39 & 39 & & & \\
\hline benign tumor & & & & & & & & & & 48 & $22-75$ & 57 & 56.8 & 23 & 25 & & & \\
\hline Lung inflammation & & & & & & & & & & 19 & $47-68$ & 55 & 55.6 & 12 & 7 & & & \\
\hline pulmonary tuberculosis & & & & & & & & & & 11 & $26-75$ & 57 & 57.5 & 4 & 7 & & & \\
\hline Lung cancer & 350 & $27-85$ & 60 & 59.6 & 218 & 132 & 140 & 169 & 41 & 411 & $28-87$ & 60 & 59.9 & 267 & 144 & 181 & 161 & 69 \\
\hline stageI & 95 & $27-76$ & 61 & 61.1 & 58 & 37 & 21 & 47 & 27 & 123 & $28-87$ & 60 & 59.3 & 71 & 52 & 29 & 52 & 42 \\
\hline stageII & 60 & $40-85$ & 59 & 60.3 & 37 & 33 & 18 & 39 & 3 & 76 & $42-82$ & 62 & 51.8 & 51 & 25 & 31 & 33 & 12 \\
\hline stageIII & 100 & $38-80$ & 60 & 59.6 & 66 & 34 & 48 & 49 & 3 & 112 & $38-86$ & 59 & 60.1 & 80 & 32 & 64 & 43 & 5 \\
\hline stageIV & 95 & $36-78$ & 57 & 57.5 & 57 & 38 & 53 & 34 & 8 & 100 & $35-78$ & 59 & 58.7 & 68 & 32 & 57 & 33 & 10 \\
\hline NSCLC & 319 & $27-80$ & 60 & 59.6 & 195 & 124 & 110 & 168 & 41 & 371 & $28-87$ & 60 & 59.7 & 235 & 136 & 141 & 161 & 69 \\
\hline squamous cell carcinoma & 114 & $27-80$ & 62 & 61.1 & 90 & 24 & 44 & 65 & 5 & 114 & $28-86$ & 62 & 61.5 & 99 & 15 & 51 & 60 & 3 \\
\hline adenocarcinoma & 197 & $33-78$ & 59 & 58.9 & 101 & 96 & 62 & 100 & 35 & 247 & $32-87$ & 59 & 58.8 & 128 & 119 & 84 & 87 & 76 \\
\hline other & 8 & $37-69$ & 57 & 56.4 & 4 & 4 & 4 & 3 & 1 & 10 & $38-74$ & 62 & 60.3 & 8 & 2 & 6 & 4 & 0 \\
\hline SCLC & 31 & $42-85$ & 58 & 59.1 & 23 & 8 & 30 & 1 & 0 & 40 & $46-82$ & 62 & 61.4 & 32 & 8 & 40 & 0 & 0 \\
\hline
\end{tabular}

Medical Sciences (CICAMS, Beijing, China). Patients with lung cancer and benign disease were confirmed by histopathological analysis (Lung puncture, bronchoscopy sampling or surgery), according to the criteria defined by the American Joint Committee on Cancer. Healthy subjects were confirmed by their negative results in $\mathrm{X}$-ray, ultrasound and CT examination. The clinical characteristics of the subjects are listed in Table 2. Blood samples were obtained from all study cases involved in our present study. For all lung cancer patients, samples were obtained prior to first treatment.

The characterization and validation of serum MIC-1 and its combination were divided into 2 study phases. In phase 1 (training) study, serum MIC-1 and other biomarkers were tested and evaluated for lung cancer in training group, and a probability algorithm was generated by logistic regression. In the phase 2 (validation) study, serum samples were evaluated independently for confirmation of diagnostic parameters and cross-validation of the probability algorithm model in the validation group (supplementary Figure 1). The study has been approved by the Ethics Committee of CICAMS.

\section{Serum MIC-1 detection by in-house ELISA method}

Serum MIC-1 was detected by a sensitive ELISA, which produced by CICAMS as detailed previously $[37,38]$. All samples were analyzed using ELISA assays on the same day according to the "Standards for the reporting of diagnostic accuracy studies (STARD) initiative" (Supplementary Table 7). Each test included two standard control $(\mathrm{CV}<12 \%)$. All serum samples were duplicately assayed.

\section{Serum CEA, CA125, NSE, SCC and Cyfra21-1 assay}

We detected a panel of five biomarkers, namely CA125, CEA, NSE, Cyfra21-1and SCC. The serum levels of CEA, CA125, NSE and Cyfra21-1 were tested by electrochemiluminescence immunoassay (ECLIA) kits using Elecsys 2010 (Roche, America). The levels of SCC in the serum were detected by SCC assay kit using an ARCHITECT I2000SR immune analyze system (Abbott, America). Each test included a standard control $(\mathrm{CV}<5 \%)$.

\section{Statistics}

The Mann-Whitney test and Kruskal-Wallis test were performed to measure the serum MIC-1 between unpaired groups and among all groups, respectively. ROC curve was used to identify the diagnostic information. Multivariable logistic regression model was conducted to corporate diagnostic performance of biomarkers. The clinical cut-off value for MIC-1 was assigned as the mean value plus two point five standard deviations of healthy individuals, and the clinical cut-off values for CEA, CA125, NSE, Cyfra21-1 and SCC were based on the manufacturer's protocols. The statistical activity was operated with the Statistical Package for the Social Sciences, version 19.0 (SPSS). $P$ value of $<0.05$ for a twosided test was considered to be statistically significant. 


\section{Abbreviations}

MIC-1: Macrophage inhibitory cytokine 1; LDCT: Low Dosage Computerized Tomography; ROC: Receiver operating characteristic.

\section{CONFLICTS OF INTEREST}

The authors declare that they have no competing interests.

\section{FUNDING}

This work was supported by National Natural Science Foundation of China (81441080; 81502023), National High Technology Research and Development Program (2008AA02Z415), Capital Clinical Application characteristic Research (Z121107001012066) and Scholarship Program from China Scholarship Council (201508110083).

\section{REFERENCES}

1. Siegel RL, Miller KD, Jemal A. Cancer statistics, 2016. CA Cancer J Clin. 2016; 66:7-30.

2. Goldstraw P, Crowley J, Chansky K, Giroux DJ, Groome PA, Rami-Porta R, Postmus PE, Rusch V, Sobin L, International Association for the Study of Lung Cancer International Staging C, Participating I. The IASLC Lung Cancer Staging Project: proposals for the revision of the TNM stage groupings in the forthcoming (seventh) edition of the TNM Classification of malignant tumours. J Thorac Oncol. 2007; 2:706-14.

3. International Early Lung Cancer Action Program I, Henschke CI, Yankelevitz DF, Libby DM, Pasmantier MW, Smith JP, Miettinen OS. Survival of patients with stage I lung cancer detected on CT screening. N Engl J Med. 2006; 355:1763-71.

4. National Lung Screening Trial Research T, Church TR, Black WC, Aberle DR, Berg CD, Clingan KL, Duan F, Fagerstrom RM, Gareen IF, Gierada DS, Jones GC, Mahon I, Marcus PM, et al. Results of initial low-dose computed tomographic screening for lung cancer. N Engl J Med. 2013; 368:1980-91.

5. Tarro G, Perna A, Esposito C. Early diagnosis of lung cancer by detection of tumor liberated protein. J Cell Physiol. 2005; 203:1-5.

6. Hiley CT, Le Quesne J, Santis G, Sharpe R, de Castro DG, Middleton G, Swanton C. Challenges in molecular testing in non-small-cell lung cancer patients with advanced disease. Lancet. 2016; 388:1002-11.

7. Sturgeon CM, Duffy MJ, Stenman UH, Lilja H, Brünner N, Chan DW, Babaian R, Bast RC Jr, Dowell B, Esteva FJ, Haglund C, Harbeck N, Hayes DF, et al. National Academy of Clinical Biochemistry laboratory medicine practice guidelines for use of tumor markers in testicular, prostate, colorectal, breast, and ovarian cancers. Clin Chem. 2008; 54:e11-79.

8. Plebani M, Basso D, Navaglia F, De Paoli M, Tommasini A, Cipriani A. Clinical evaluation of seven tumour markers in lung cancer diagnosis: can any combination improve the results? Br J Cancer. 1995; 72:170-3.

9. Sun M, Song J, Zhou Z, Zhu R, Jin H, Ji Y, Lu Q, Ju H. Comparison of Serum MicroRNA21 and Tumor Markers in Diagnosis of Early Non-Small Cell Lung Cancer. Dis Markers. 2016; 2016:3823121.

10. Patz EF Jr, Campa MJ, Gottlin EB, Kusmartseva I, Guan XR, Herndon JE 2nd. Panel of serum biomarkers for the diagnosis of lung cancer. J Clin Oncol. 2007; 25:5578-83.

11. Li X, Asmitananda T, Gao L, Gai D, Song Z, Zhang Y, Ren H, Yang T, Chen T, Chen M. Biomarkers in the lung cancer diagnosis: a clinical perspective. Neoplasma. 2012; 59:500-7.

12. Tan HT, Low J, Lim SG, Chung MC. Serum autoantibodies as biomarkers for early cancer detection. FEBS J. 2009; 276:6880-904.

13. Qiu J, Choi G, Li L, Wang H, Pitteri SJ, Pereira-Faca SR, Krasnoselsky AL, Randolph TW, Omenn GS, Edelstein C, Barnett MJ, Thornquist MD, Goodman GE, et al. Occurrence of autoantibodies to annexin I, 14-3-3 theta and LAMR1 in prediagnostic lung cancer sera. J Clin Oncol. 2008; 26:5060-6.

14. Madoz-Gurpide J, Kuick R, Wang H, Misek DE, Hanash SM. Integral protein microarrays for the identification of lung cancer antigens in sera that induce a humoral immune response. Mol Cell Proteomics. 2008; 7:268-81.

15. Bootcov MR, Bauskin AR, Valenzuela SM, Moore AG, Bansal M, He XY, Zhang HP, Donnellan M, Mahler S, Pryor K, Walsh BJ, Nicholson RC, Fairlie WD, et al. MIC-1, a novel macrophage inhibitory cytokine, is a divergent member of the TGF-beta superfamily. Proc Natl Acad Sci USA. 1997; 94:11514-9.

16. Fairlie WD, Moore AG, Bauskin AR, Russell PK, Zhang HP, Breit SN. MIC-1 is a novel TGF-beta superfamily cytokine associated with macrophage activation. J Leukoc Biol. 1999; 65:2-5.

17. Paralkar VM, Vail AL, Grasser WA, Brown TA, Xu H, Vukicevic S, Ke HZ, Qi H, Owen TA, Thompson DD. Cloning and characterization of a novel member of the transforming growth factor-beta/bone morphogenetic protein family. J Biol Chem. 1998; 273:13760-7.

18. Buckhaults P, Rago C, St Croix B, Romans KE, Saha S, Zhang L, Vogelstein B, Kinzler KW. Secreted and cell surface genes expressed in benign and malignant colorectal tumors. Cancer Res. 2001; 61:6996-7001.

19. Brown DA, Ward RL, Buckhaults P, Liu T, Romans KE, Hawkins NJ, Bauskin AR, Kinzler KW, Vogelstein B, 
Breit SN. MIC-1 serum level and genotype: associations with progress and prognosis of colorectal carcinoma. Clin Cancer Res. 2003; 9: 2642-50.

20. Boyle GM, Pedley J, Martyn AC, Banducci KJ, Strutton GM, Brown DA, Breit SN, Parsons PG. Macrophage inhibitory cytokine-1 is overexpressed in malignant melanoma and is associated with tumorigenicity. J Invest Dermatol. 2009; 129: 383-91.

21. Wang X, Li Y, Tian H, Qi J, Li M, Fu C, Wu F, Wang Y, Cheng D, Zhao W, Zhang C, Wang T, Rao J, et al. Macrophage inhibitory cytokine 1 (MIC-1/GDF15) as a novel diagnostic serum biomarker in pancreatic ductal adenocarcinoma. BMC Cancer. 2014; 14: 578.

22. Huh SJ, Chung CY, Sharma A, Robertson GP. Macrophage inhibitory cytokine-1 regulates melanoma vascular development. Am J Pathol. 2010; 176: 2948-57.

23. Mimeault M, Batra SK. Divergent molecular mechanisms underlying the pleiotropic functions of macrophage inhibitory cytokine-1 in cancer. J Cell Physiol. 2010; 224:626-35.

24. Senapati S, Rachagani S, Chaudhary K, Johansson SL, Singh RK, Batra SK. Overexpression of macrophage inhibitory cytokine-1 induces metastasis of human prostate cancer cells through the FAK-RhoA signaling pathway. Oncogene. 2010; 29:1293-302.

25. McWilliams AM, Mayo JR, Ahn MI, MacDonald SL, Lam SC. Lung cancer screening using multi-slice thinsection computed tomography and autofluorescence bronchoscopy. J Thorac Oncol. 2006; 1:61-8.

26. Bruzzese F, Hagglof C, Leone A, Sjoberg E, Roca MS, Kiflemariam S, Sjoblom T, Hammarsten P, Egevad L, Bergh A, Ostman A, Budillon A, Augsten M. Local and systemic protumorigenic effects of cancer-associated fibroblast-derived GDF15. Cancer Res. 2014; 74:3408-17.

27. Karan D, Holzbeierlein J, Thrasher JB. Macrophage inhibitory cytokine-1: possible bridge molecule of inflammation and prostate cancer. Cancer Res. 2009; 69:2-5.

28. Breit SN, Johnen H, Cook AD, Tsai VW, Mohammad MG, Kuffner T, Zhang HP, Marquis CP, Jiang L, Lockwood G, Lee-Ng M, Husaini Y, Wu L, et al. The TGF-beta superfamily cytokine, MIC-1/GDF15: a pleotrophic cytokine with roles in inflammation, cancer and metabolism. Growth Factors. 2011; 29:187-95.

29. Chen SJ, Karan D, Johansson SL, Lin FF, Zeckser J, Singh AP, Batra SK, Lin MF. Prostate-derived factor as a paracrine and autocrine factor for the proliferation of androgen receptor-positive human prostate cancer cells. Prostate. 2007; 67:557-71.

30. Jin YJ, Lee JH, Kim YM, Oh GT, Lee H. Macrophage inhibitory cytokine-1 stimulates proliferation of human umbilical vein endothelial cells by up-regulating cyclins D1 and E through the PI3K/Akt-, ERK-, and JNK-dependent AP-1 and E2F activation signaling pathways. Cell Signal. 2012; 24:1485-95.

31. Molina R, Auge JM, Escudero JM, Marrades R, Vinolas N, Carcereny E, Ramirez J, Filella X. Mucins CA 125, CA 19.9, CA 15.3 and TAG-72.3 as tumor markers in patients with lung cancer: comparison with CYFRA 21-1, CEA, SCC and NSE. Tumour Biol. 2008; 29:371-80.

32. Shacter E, Weitzman SA. Chronic inflammation and cancer. Oncology (Williston Park). 2002; 16:217-29.

33. Novello S, Fava C, Borasio P, Dogliotti L, Cortese G, Crida B, Selvaggi G, Lausi P, Brizzi MP, Sperone P, Cardinale L, Ferraris F, Perotto F, et al. Three-year findings of an early lung cancer detection feasibility study with lowdose spiral computed tomography in heavy smokers. Ann Oncol. 2005; 16:1662-6.

34. Ebbert JO, Yang P, Vachon CM, Vierkant RA, Cerhan JR, Folsom AR, Sellers TA. Lung cancer risk reduction after smoking cessation: observations from a prospective cohort of women. J Clin Oncol. 2003; 21:921-6.

35. Sobue T, Yamamoto S, Hara M, Sasazuki S, Sasaki S, Tsugane S, Center JSGJPH. Cigarette smoking and subsequent risk of lung cancer by histologic type in middleaged Japanese men and women: the JPHC study. Int J Cancer. 2002; 99:245-51.

36. Pannala R, Basu A, Petersen GM, Chari ST. New-onset diabetes: a potential clue to the early diagnosis of pancreatic cancer. Lancet Oncol. 2009; 10:88-95.

37. Wang XB, Jiang XR, Yu XY, Wang L, He S, Feng FY, Guo LP, Jiang W, Lu SH. Macrophage inhibitory factor 1 acts as a potential biomarker in patients with esophageal squamous cell carcinoma and is a target for antibody-based therapy. Cancer Sci. 2014; 105:176-85.

38. Wang X, Yang Z, Tian H, Li Y, Li M, Zhao W, Zhang C, Wang T, Liu J, Zhang A, Shen D, Zheng C, Qi J, et al. Circulating MIC-1/GDF15 is a complementary screening biomarker with CEA and correlates with liver metastasis and poor survival in colorectal cancer. Oncotarget. 2017; 8:24892-24901. doi: 10.18632/oncotarget.15279. 